\title{
The treatment of sexually transmitted infections
}

\author{
Armorel D van Eyk \\ Division of Pharmacology, Department of Pharmacy and Pharmacology, School of Therapeutic Sciences \\ Faculty of Health Sciences, University of Witwatersrand, Johannesburg
}

*Corresponding author, email: Armorel.vanEyk@wits.ac.za

\begin{abstract}
The high level of sexually transmitted infections (STIs) in South Africa can be attributed to a large number of factors including low socio-economic conditions, social stigma, gender inequalities, inability to access adequate health care systems and lack of preventative programmes. The main curable STIs consist of chlamydia, gonorrhoea, syphilis and trichomoniasis, most of which occur in the developing world. The inability to adequately treat the infections leads to morbidity and has wide-ranging consequences on reproductive health and the health of infants. Due to the inefficient treatment of STIs, the World Health Organisation (WHO) recommended and promoted the syndromic management of STIs in developing countries at the point of contact with the health service provider with the intention of improving the quality of care of these patients. The syndromic approach to STI management depends on patient symptoms and the signs presented at the clinical examination. The four main syndrome management protocols are for urethral discharge and swollen testes, genital ulcers, vaginal discharge and lower abdominal pain in women. After identification of a syndrome, combined therapy is utilised to treat the common causes of the infection. A diagnosis can be made quite rapidly without the need for expensive equipment and tests or special skills. Health care providers make use of flow charts or algorithms for diagnosis and treatment. Algorithms or flowcharts should be adapted to local conditions taking into consideration aetiology, local antimicrobial susceptibility patterns and drug availability. These protocols will help with the prevention and treatment STIs in South Africa.
\end{abstract}

Keywords: Sexually transmitted infections; Chlamydia; Gonorrhoea, Syphilis; Syndromic Management

\section{Defining a sexually transmitted infection (STI)}

Sexually transmitted infections are infections caused by bacteria, viruses and parasites that are transferred mainly via sexual contact, be it vaginal, anal, oral or in some instances via non-sexual means, i.e. by means of blood or blood products. Mother-to-child transmission of e.g. chlamydia, gonorrhoea, syphilis, hepatitis B and HIV can also occur during pregnancy and childbirth. ' A large number of factors, including socio-economic and gender inequalities as well as preventative program failure and inaccessibility to adequate health services have contributed to the epidemic of classic STIs. ${ }^{2}$ In South Africa, diagnostic tests for STIs are for the majority of patients expensive, geographically inaccessible and often time consuming, in that they have to wait a long time to receive results. This may result in the treatment being incomplete and failing. Rapid tests for syphilis and HIV are available while tests for other STIs are under development. ${ }^{1}$

\section{Most common bacterial STIs and their treatment}

\section{Chlamydia}

Chlamydial infections are the most common STIs, occurring predominantly in young sexually active adults. The bacterium
Chlamydia trachomatis is responsible for the infection. It causes cervicitis in females and urethritis in males as well as rectal and oropharyngeal infections. Although a chlamydia infection does not display any symptoms in many patients, pain during urination as well as a discharge or vaginal bleeding has been noted. ${ }^{3}$ There are various tests that can be utilized for diagnosis, e.g. culture, enzyme-linked immunosorbent assays (ELISAs), direct immunofluorescence assays (DFAs) and nucleic acid amplification tests (NAATs). The latter test is preferred due to its superior performance. A strong recommendation is that updated global guidance must be taken into account, due to the establishment of standardised national protocols. These guidelines must then be adapted to local epidemiological conditions and microbial resistance patterns. The WHO recommended treatment of uncomplicated genital chlamydia is azithromycin ( $1 \mathrm{~g}$ orally, single dose) preferred to doxycycline (100 mg orally, twice daily for 7 days). Doxycycline is preferred in anorectal chlamydial infections (7 days) and lymphogranuloma venereum in homosexual men, and HIV infected patients (21 days). For neonatal conjunctivitis azithromycin is also preferred (20 $\mathrm{mg} / \mathrm{kg}$ orally for 3 days). ${ }^{3}$ 


\section{Gonorrhoea}

Gonorrhoea is the second most common STI, caused by the bacterium Neisseria gonorrhoeae, with discharge and painful urination both symptoms of this infection. It is often asymptomatic in women and if left untreated may lead to serious complications, i.e. pelvic inflammatory disease, ectopic pregnancy as well as infertility. In men untreated infections may lead to epididymitis, urethral stricture and infertility. Neonatal conjunctivitis in infants, which may result in blindness, is transferred from mothers that have the infection. This infection can be diagnosed clinically by vaginal and urethral discharge or in countries with laboratory support by using culturing or nucleic acid amplification tests (NAATs). ${ }^{4}$ Due to the rapid changing antimicrobial susceptibility patterns for gonorrhoea, treatment is challenging and may eventually lead to untreatable infections. Treatment recommendations have to be constantly updated due to the changing drug resistance patterns, e.g. first line treatment using quinolones and third generation cephalosporins has led to increasing treatment failures in various countries. Updated global guidance must be taken into account, due to the establishment of standardised national protocols. Local epidemiological conditions and microbial resistance patterns must be considered before treatment. ${ }^{5-16}$ The WHO recommended treatment of genital, oropharyngeal and anorectal gonorrhoea is dual therapy consisting of either ceftriaxone ( $250 \mathrm{mg} I \mathrm{M}$, single dose) plus azithromycin ( $1 \mathrm{~g}$ orally, single dose) or cefixime (400 mg orally, single dose) plus azithromycin (1 g orally, single dose)

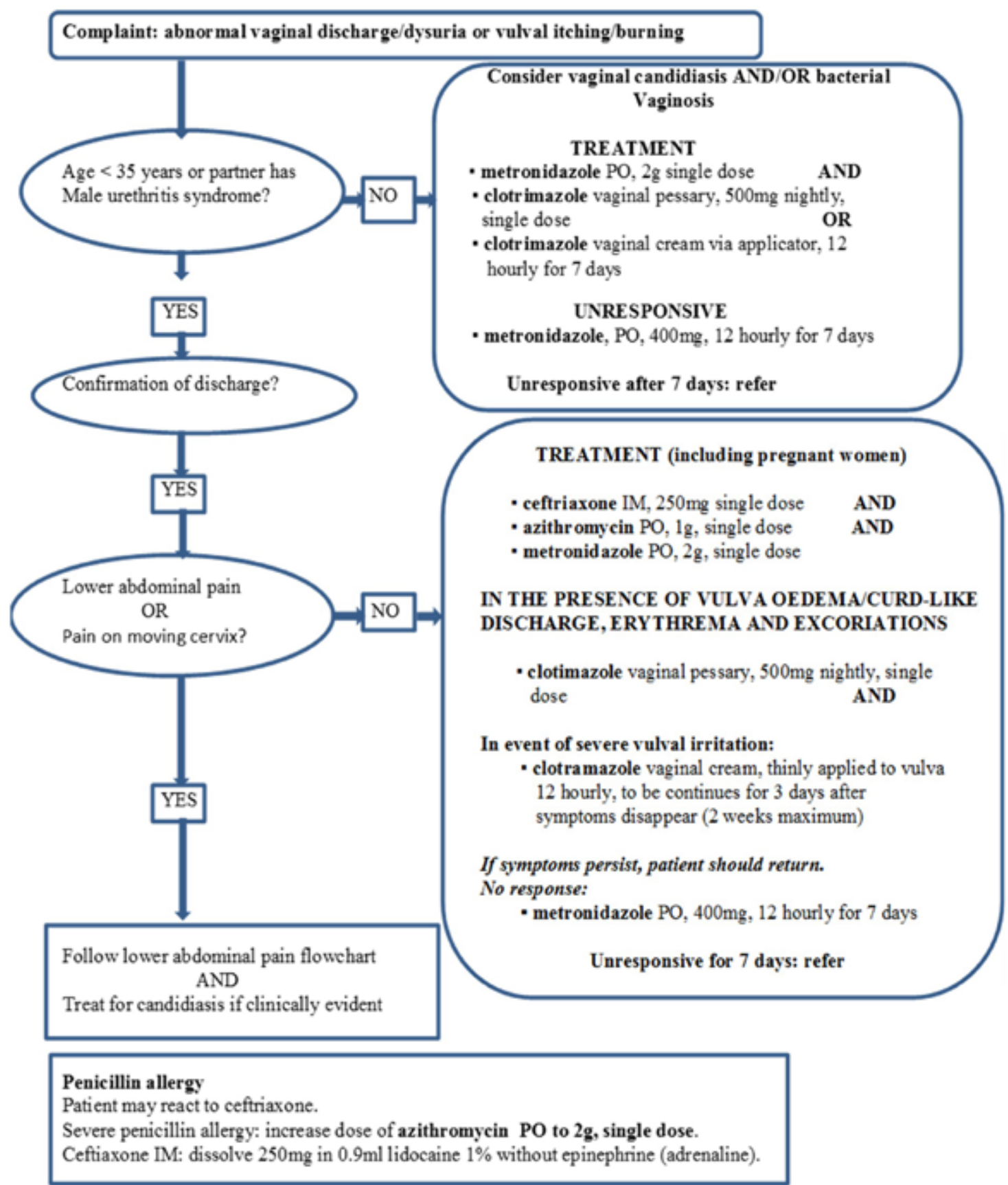

Figure 1: Flowchart of syndromic treatment of Vaginal Discharge Syndrome. (adapted from the revised VDS flowchart of the Primary Health Care Standard Treatment Guidelines and Essential Drugs List, 2015) ${ }^{23}$ 
over single therapy (ceftriaxone $250 \mathrm{mg} I \mathrm{M}$, single dose; cefixime $400 \mathrm{mg}$ orally, single dose or spectinomycin $2 \mathrm{~g} \mathrm{IM}$, single dose). For neonatal conjunctivitis either ceftriaxone, kanamycin or spectinomycin is effective. For further details on dosages and retreatment, refer to the WHO treatment guidelines. ${ }^{4}$

\section{Syphilis}

Syphilis is caused by the bacterium Treponema pallidum and initially displays as a painless sore on the genitals or around the mouth (4-6 weeks) followed by a rash and a sore throat (a few weeks). Treatment is quite easy, however untreated syphilis may lead to blindness, stroke, paralysis and death. This infection is transmitted sexually via contact with infected membranes and abraded skin. It can also be transmitted via blood transfusions or from mother to foetus via the placenta. The diagnosis of the infection depends on clinical history, physical examination, radiology and laboratory testing. Various serologic tests are used for diagnosis, while much more rapid tests are currently also available for syphilis only or dual HIV/ syphilis. ${ }^{17}$ The WHO recommended treatment of early syphilis is benzathine penicillin $\mathrm{G}$ (2.4 million units IM, once-off). For late syphilis, benzathine penicillin $\mathrm{G}$ once-weekly for 3 consecutive weeks is recommended. ${ }^{17}$ For congenial syphilis aqueous benzyl penicillin (IV) is recommended. For further details on dosages and retreatment, refer to the WHO treatment guidelines. ${ }^{17}$

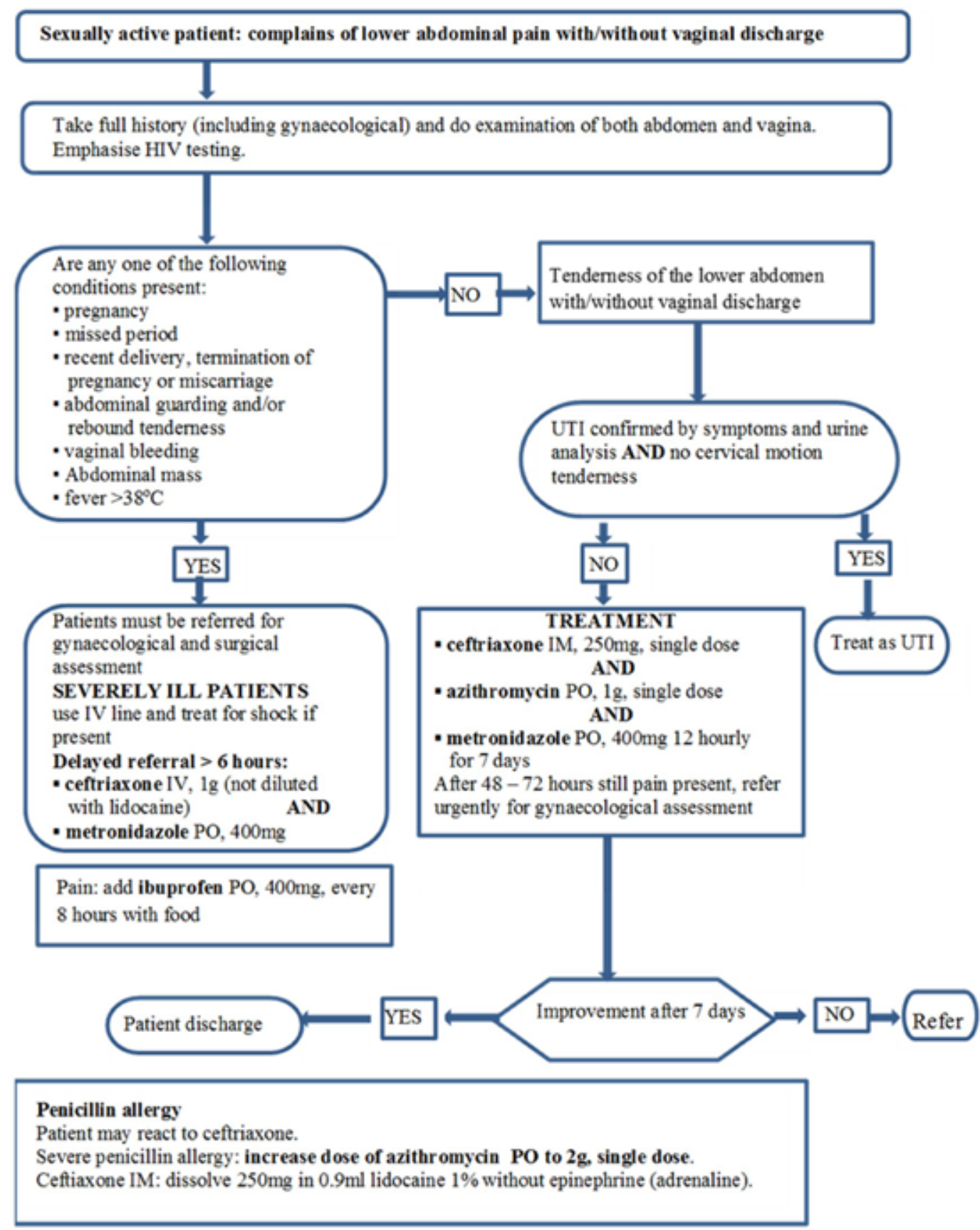

Figure 2: Flowchart of syndromic treatment of Lower Abdominal Pain. (adapted from the revised LAP flowchart of the Primary Health Care Standard Treatment Guidelines and Essential Drugs List, 2015) ${ }^{23}$ 


\section{Syndromic treatment of STIs}

In developing countries like South Africa, syndromic treatment and management of STIs are followed at initial point of contact with health services. Health workers thus rely on the diagnosis of a specific syndrome by taking note of observed clinical signs and symptoms patients complain of and by making use of clinical algorithms or flow charts. Examples of observed syndromes include genital ulcers, abdominal pain, vaginal discharge and urethral discharge. This approach to STI treatment is rapid and simple with on-the-day treatment. An unfortunate disadvantage of this approach is that STIs that show no syndromes are missed. After identification of a particular STI syndrome, a combination therapy is followed for treatment. For local situations, local data on the causes of a syndrome, pathogen susceptibility to antimicrobial agents as well as available antimicrobial agents must be taken into account when following algorithms or flow charts. Important revisions of the syndromic flowcharts should constantly be undertaken, by taking cognisance of changing antibiotic resistance and coinfection patterns. ${ }^{2,13,18-23}$

\section{Local factors responsible for revision in syndromic flowcharts}

\section{Ciprofloxacin-resistant gonorrhoea}

Ciprofloxacin as a single dose was the first line treatment of gonorrhoea in South Africa, however Neisseria gonorrhoea organisms (QRNG) have become increasingly resistant to this treatment. The only other alternative treatment options for this infection currently are the two cephalosporins, cefixime (oral) and ceftriaxone (IM). Resistance to cefixime has been

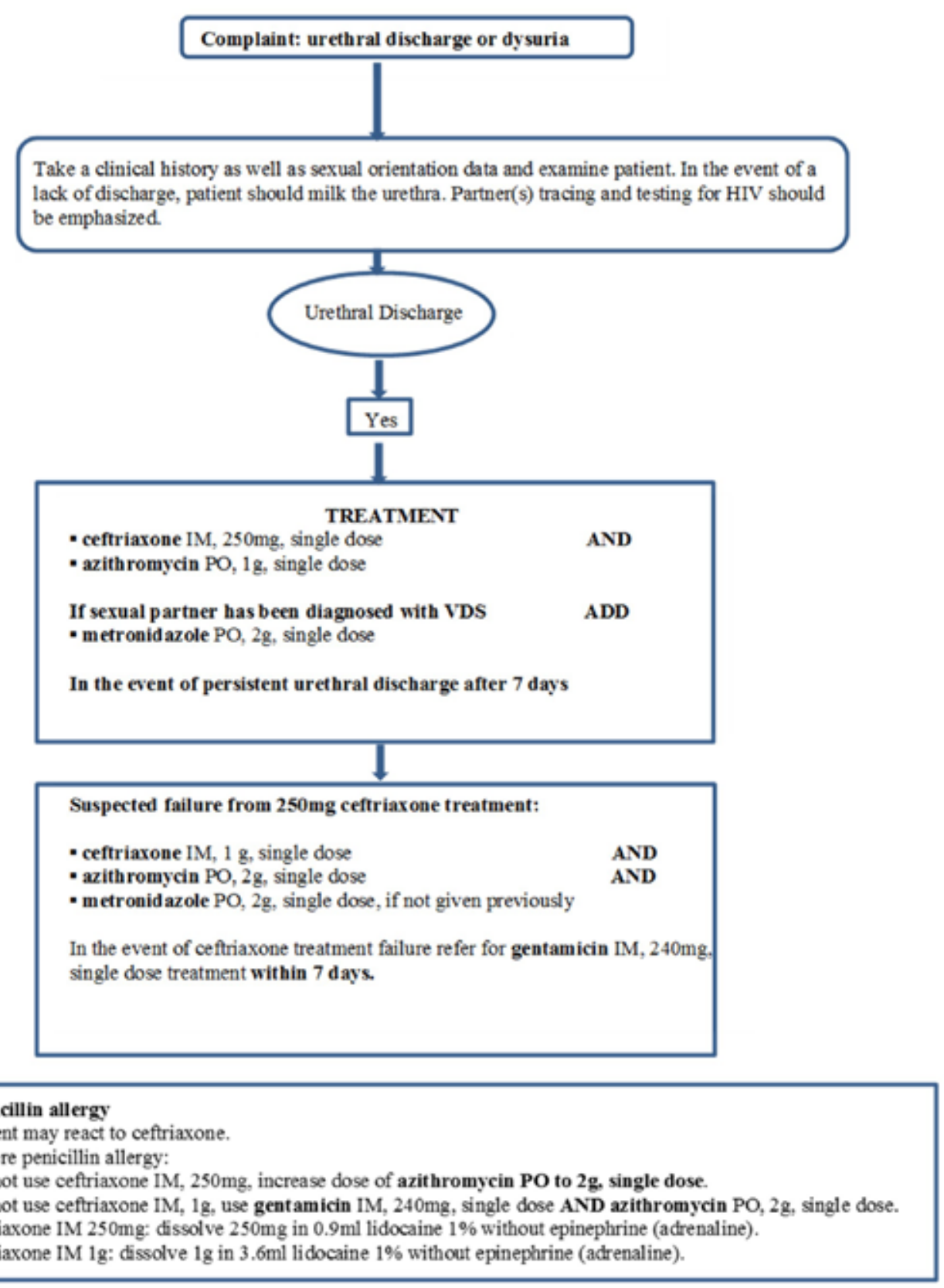

Figure 3: Flowchart of syndromic treatment of Male Urethritis Syndrome. (adapted from the revised MUS flowchart of the Primary Health Care Standard Treatment Guidelines and Essential Drugs List, 2015) ${ }^{23}$ 
documented in Japan, thus ceftriaxone is the only treatment for cefixime-resistant gonorrhoea..$^{5-16,20}$

\section{Genital ulcer aetiology changes}

The majority of genital ulcers were initially (until late 1990s) caused by bacterial infections as opposed to viral infections. The main bacterial organisms responsible for genital ulcers were Haemophilus ducreyi (chancroid) and Treponema pallidum (syphilis). Within the 1990s genital ulcers caused by the Herpes simplex virus increased in prevalence in tandem with the HIV epidemic. Since then acyclovir as treatment therapy was included in the treatment regime of genital ulcers in patients with and without HIV coinfection.,2,20,24

\section{HIV testing}

Testing for HIV remains highly recommended within all syndromic flowcharts due to the increased risk of HIV coinfection of patients with STIs. ${ }^{2,20,23}$

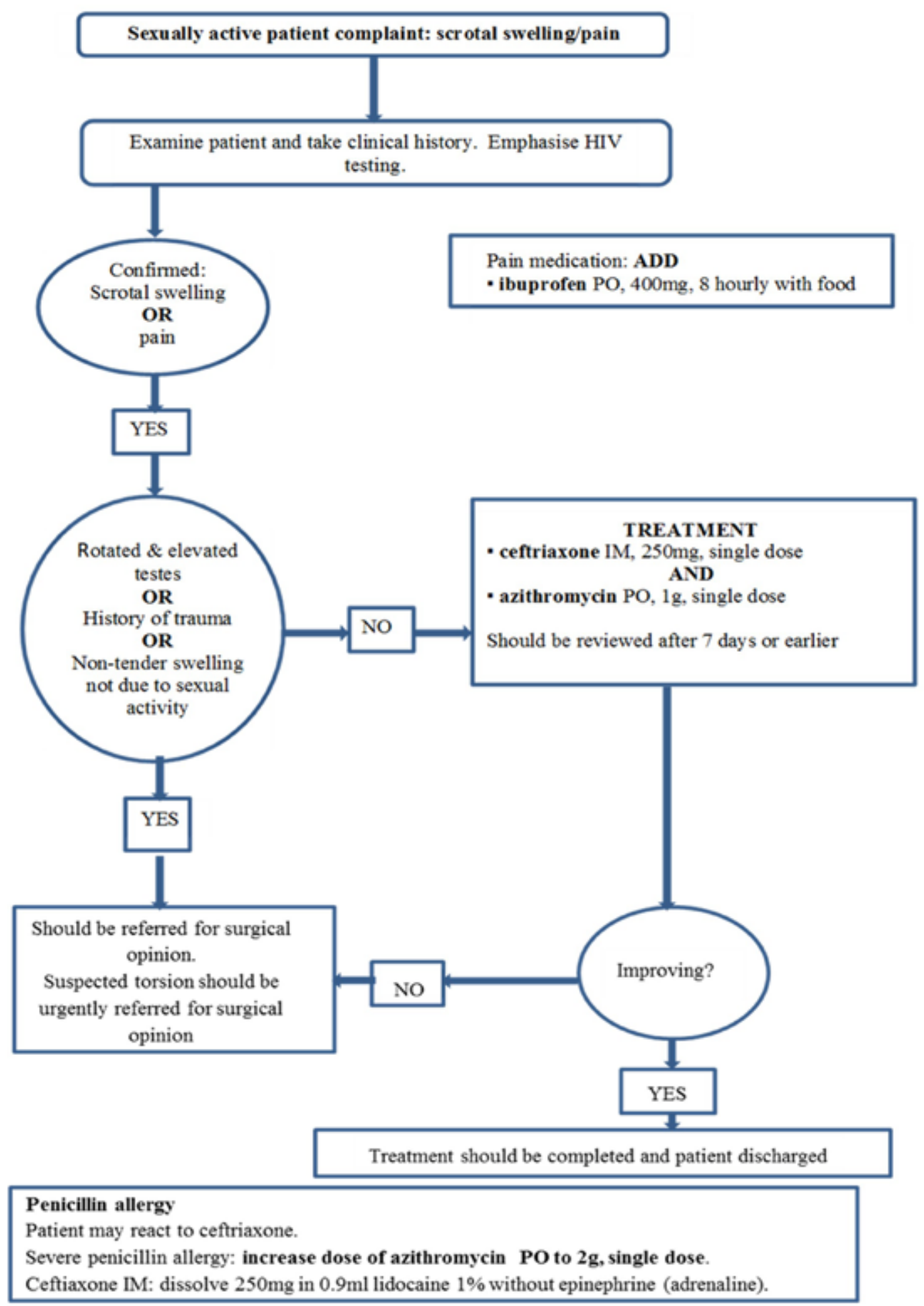

Figure 4: Flowchart of syndromic treatment of Scrotal Swelling. (adapted from the revised SSW flowchart of the Primary Health Care Standard Treatment Guidelines and Essential Drugs List, 2015) ${ }^{23}$ 


\section{Syndromic treatment flowcharts}

\section{Vaginal discharge syndrome (VDS)}

Vaginal discharge can be due to trichomoniasis, vaginosis (bacterial) and candidiasis but may also arise from N. gonorrhoea and C. trachomatis infections. The recommended protocol for VDS is presented in Figure $1 .^{23}$

\section{Lower abdominal pain (LAP)}

Pain in the lower abdominal region may be the result of pelvic inflammatory disease caused by N. gonorrhoea and C. trachomatis infections. The recommended protocol for LAP is presented in Figure $2 .{ }^{23}$

\section{Male urethritis syndrome (MUS) and scrotal swelling (SSW)}

N. gonorrhoea or C. trachomatis or a combination of both may cause urethral discharge and scrotal swelling. The recommended protocols for the treatment of each condition are presented in Figures 3 and 4, respectively. ${ }^{23}$

\section{Genital ulcer syndrome (GUS)}

The presence of genital ulcers may be due to $H$. simplex, $T$. pallidum and $H$. ducreyi or a combination of these pathogens. The recommended protocol for treatment of GUS is presented in Figure $5 .^{23}$
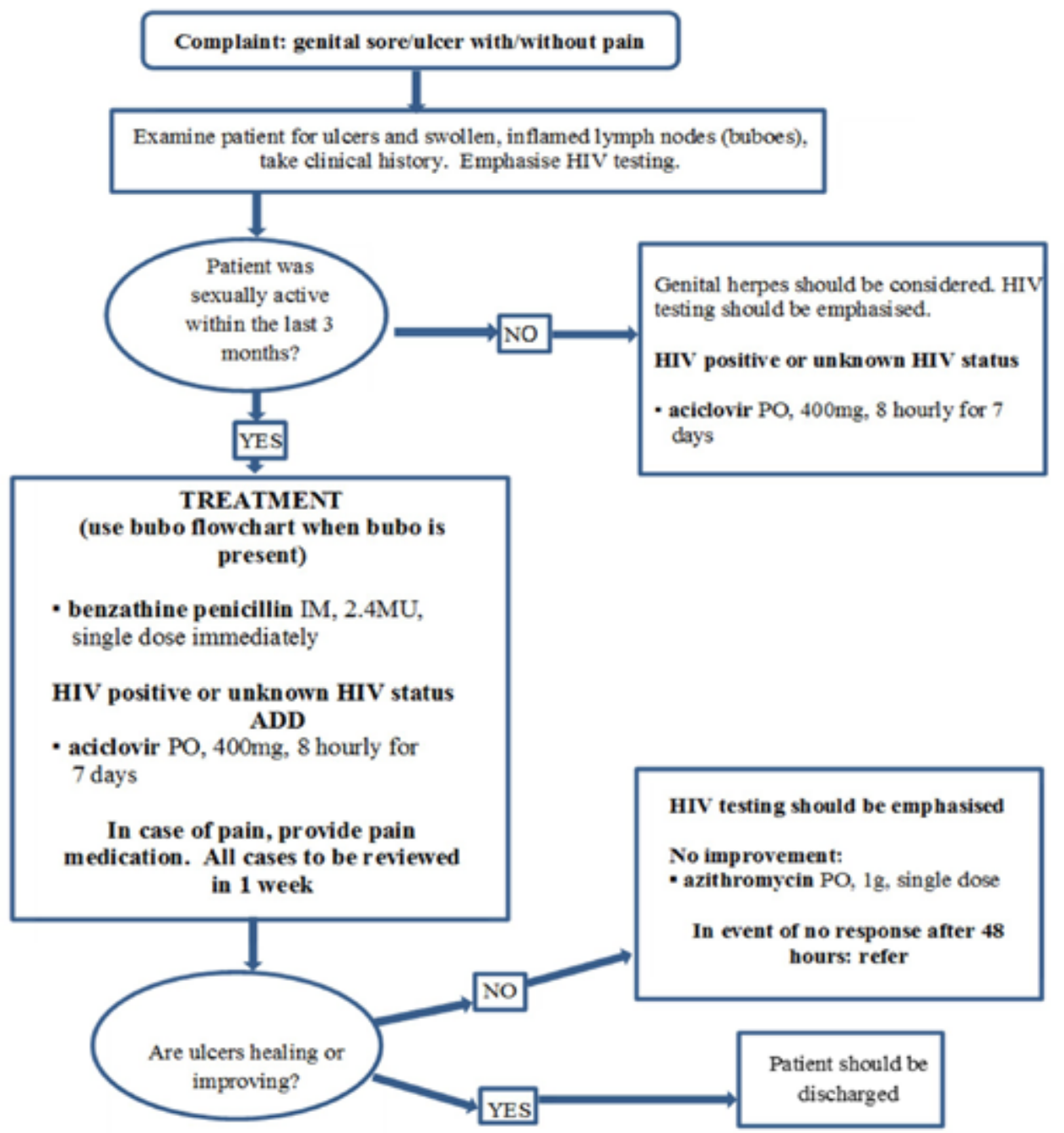

Penicillin allergy: men and non-pregnant women:

Baseline Rapid Plasmin Reagin (RPR) should be performed; benzathine penicillin should be replaced with:

- doxycycline PO, $100 \mathrm{mg}$, every 12 hours for 14 days with follow-up RPR 6 months later.

Penicillin allergy: pregnant women/breast feeding women: refer for possible penicillin-desen sitisation and confirmation of new syphilis infection.

Benzathine penicillin $\mathrm{MM}, 2.4 \mathrm{MU}: 2.4 \mathrm{MU}$ to be dissolved in $6 \mathrm{ml}$ lidocaine $1 \%$ without epinephrine (adrenaline). 


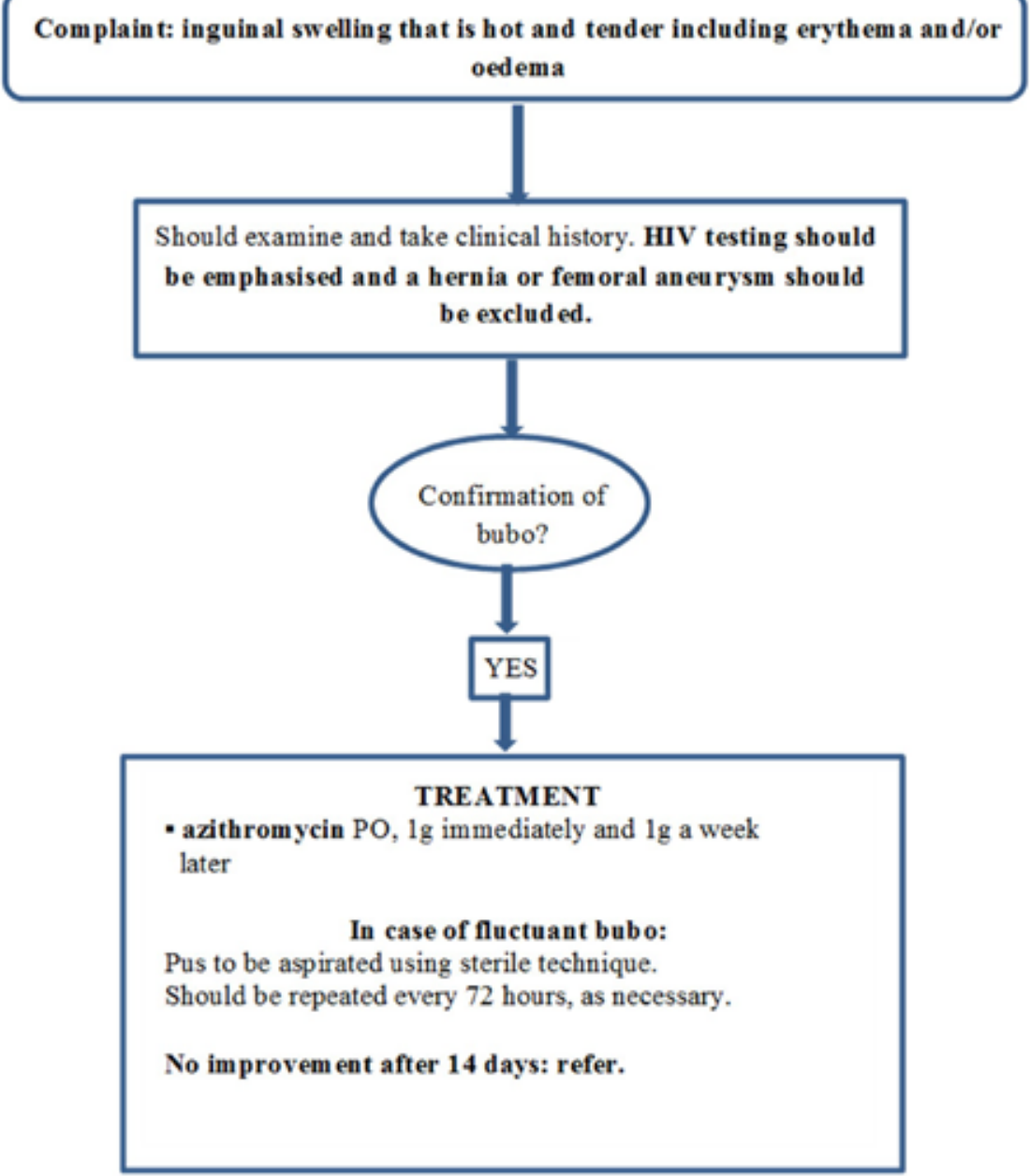

Figure 6: Flowchart of syndromic treatment of a swollen and inflamed lymph node (bubo). (adapted from the revised bubo flowchart of the Primary Health Care Standard Treatment Guidelines and Essential Drugs List, 2015)23

\section{Bubo (swollen, inflamed lymph nodes)}

A swollen and inflamed lymph node (bubo) associated with STIs usually occurs in the groin area and the pathogens responsible for this condition may be $H$. ducreyi, C. trachomatis and $N$. gonorrhoea. ${ }^{25}$ The recommended protocol for treatment of a bubo is presented in Figure $6 .{ }^{23}$

\section{Balanitis/balanoposthitis (BAL)}

An infection of the glans penis is known as balanitis and when the foreskin is involved, it is known as balanoposthitis. These conditions are more likely when urethritis is also diagnosed and may be due to STIs or non-STIs. Causative pathogens responsible for this condition include $C$. albicans as well as C. trachomatis, N. gonorrhoea, T. pallidum or H. simplex. ${ }^{26}$ The recommended protocol for treatment of a balanitis and balanoposthitis is presented in Figure 7.23

\section{Syphilis}

Syphilis can be divided into two treatment regimens: early and late syphilis. The Rapid Plasmin Reagin (RPR) can be used to measure the disease activity but it must be confirmed with a specific treponemal test. The recommended protocols for treatment of syphilis are presented in Figures 8-10.23

\section{Neonatal Conjunctivitis}

In seriously ill neonates, ceftriaxone should be used even when they are jaundiced. Calcium-containing fluids, e.g. Ringer's lactate solution, should not be given concurrently with ceftriaxone in infants $<28$ days old, however in infants $>28$ days old, ceftriaxone and calcium-containing fluids may be administered sequentially. Flushing the IV lines between administering the two products is however essential.

\section{Treatment of multiple STI syndromes}

When more than one STI syndrome is present in a patient, the treatment guidelines recommended by the National Department of Health can be followed. ${ }^{23}$

\section{Conclusion}

The syndromic management of STIs has been promoted to treat the high levels of STIs occurring in South Africa. In many rural areas, laboratory equipment and necessary skills required to diagnose specific STIs are unavailable. This approach is based 


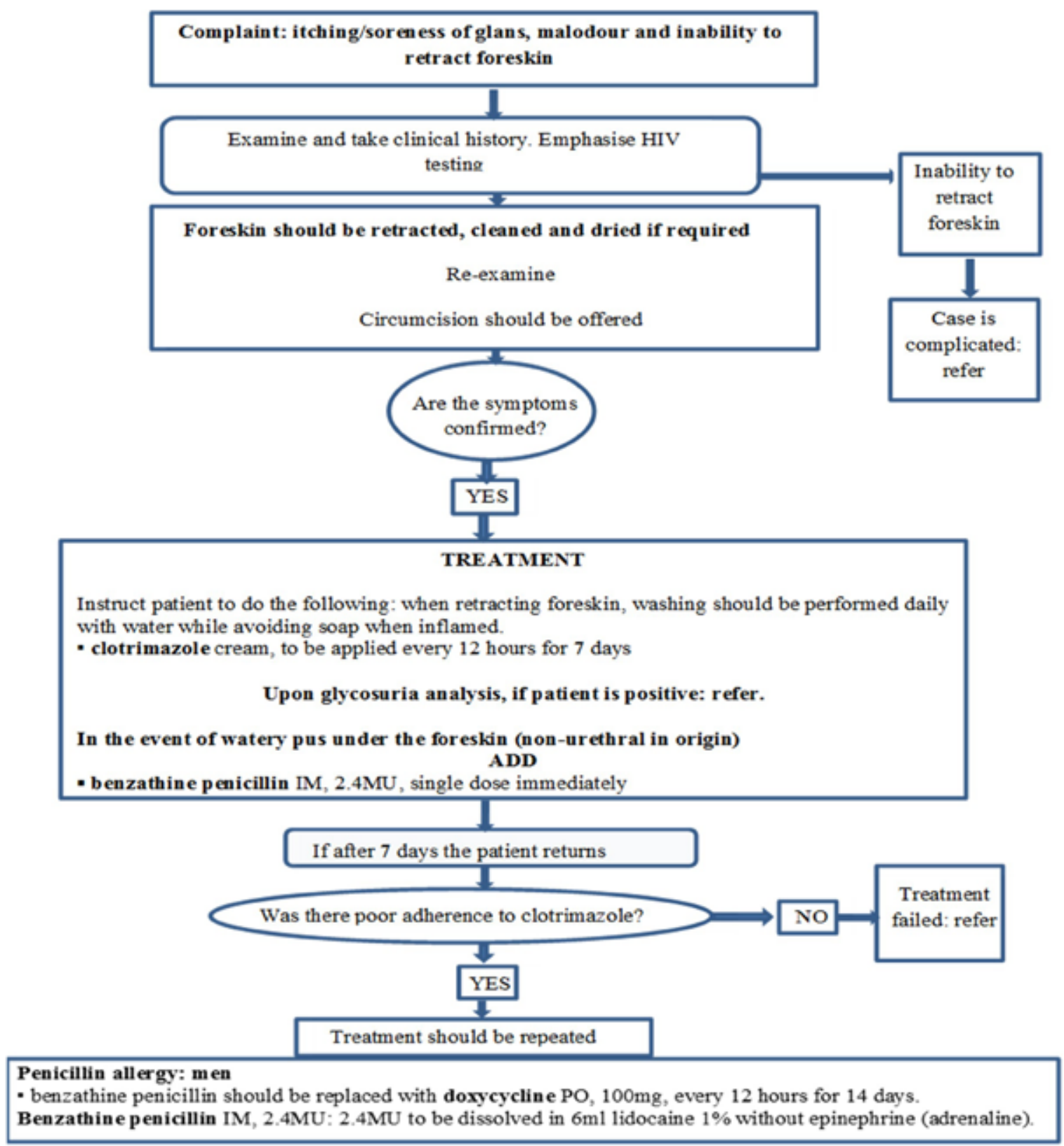

Figure 7: Flowchart of syndromic treatment of balanitis/balanoposthitis. (adapted from the revised balanitis/balanoposthitis flowchart of the Primary Health Care Standard Treatment Guidelines and Essential Drugs List, 2015) ${ }^{23}$

on the identification of symptoms and clinical signs (syndromes) that occur at the point of the first visit to the health care provider. Clinical algorithms or flowcharts are used to guide health care providers in the treatment of the organisms that usually are responsible for the syndromes. It is however very important to constantly review these tools and adapt them to local epidemiological and antimicrobial drug resistance patterns.

\section{Acknowledgements}

The author wishes to acknowledge the assistance of Prof $\mathrm{R}$ van Zyl with the proof reading of this review.

\section{References}

1. WHO. Sexually Transmitted Infections Factsheet. August 2016. Available from: http://www.who.int/mediacentre/factsheets/fs110/en/. Accessed 29 September 2016.
2. Altini L, Coetzee D. Syndromic management of sexually transmitted infections. CME. 2005 [cited];23(2):62-6. Available from: http://www.ajol.info/index.php/ cme/article/viewFile/44035/27549

3. WHO. Guidelines for the treatment of Chlamydia trachomatis. 2016 [cited]:144. Available from: https://www.ncbi.nlm.nih.gov/books/NBK379707/pdf/ Bookshelf_NBK379707.pdf

4. WHO. WHO guidelines for the treatment of Neisseria gonorrhoeae. 2016 [cited 29 September 2016];1-51. Available from: http://apps.who.int/iris/bitstr eam/10665/246114/1/9789241549691-eng.pdf

5. Moodley P, Pillay C, Goga R, Kharsany ABM, Sturm AW. Evolution in the trends of antimicrobial resistance in Neisseria gonorrhoeae isolated in Durban over a 5 year period: impact of the introduction of syndromic management. J Antimicrob Chemother. 2001; 48:853-9.

6. Crowther-Gibson P, Govender N, Lewis DA, Bamford C, Brink A, von Gottberg A et al. Human infections and antibiotic resistance. S Afr Med J. 2011 [cit]; 101(8). Available from: http://www.samj.org.za/index.php/samj/rt/ printerFriendly/5102/3367 


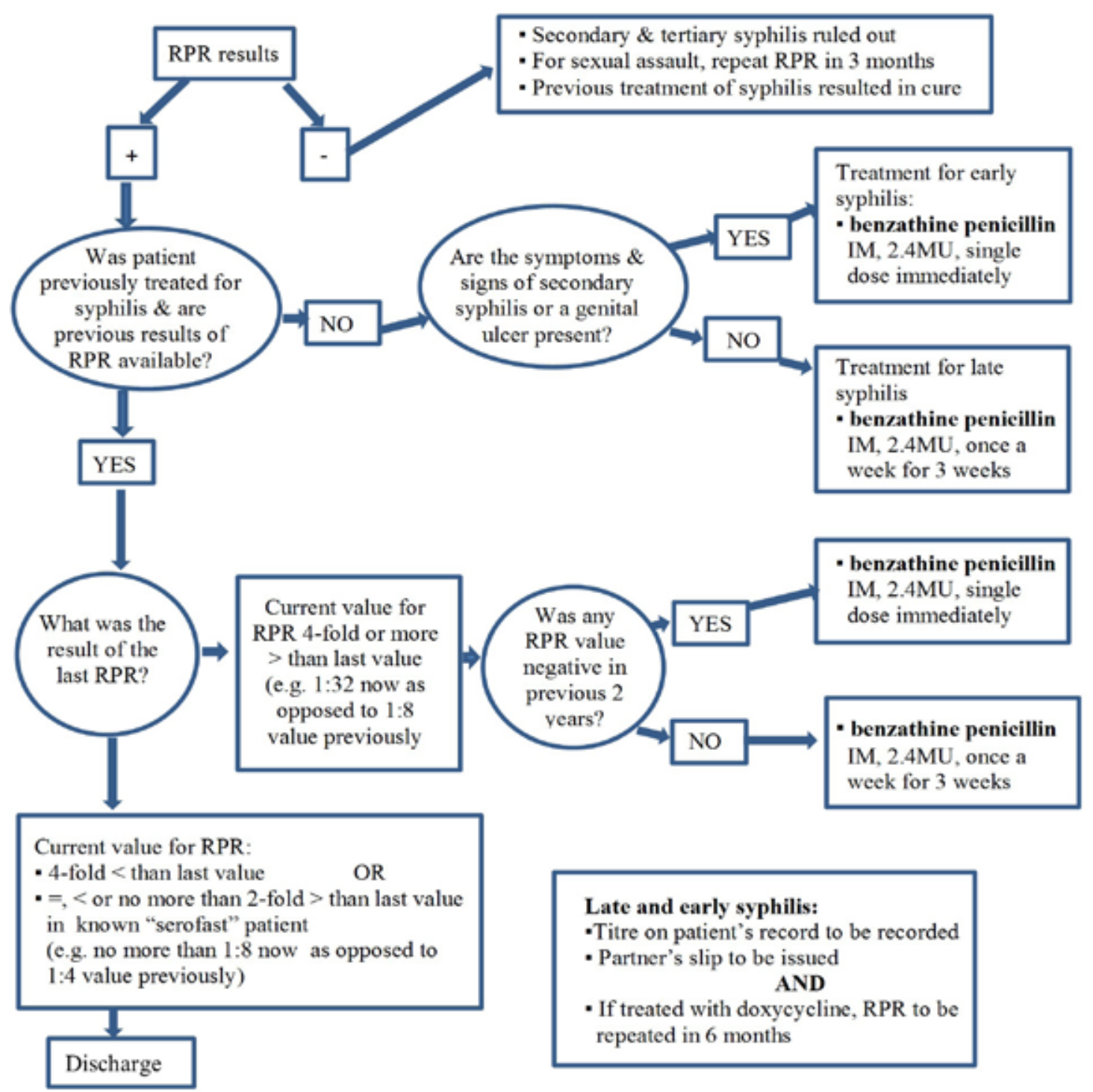

Benzathine penicillin IM, 2.4MU: $2.4 \mathrm{MU}$ to be dissolved in $6 \mathrm{ml}$ lidocaine $1 \%$ without epinephrine (adrenaline).

Figure 8: Flowchart of syndromic treatment of syphilis. (adapted from the revised syphilis flowchart of the Primary Health Care Standard Treatment Guidelines and Essential Drugs List, 2015) ${ }^{23}$

7. Lewis DA. Antimicrobial-resistant gonorrhoea in Africa: An important public health threat in need of a regional gonococcal antimicrobial surveillance programme. South Afr J Epidemiol Infect. 2011;26(4):215-20.

8. Lewis DA, Lukehart SA. Antimicrobial resistance in Neisseria gonorrhoeae and Treponema pallidum: evolution, therapeutic challenges and the need to strengthen global surveillance. Sex Transm Infect. 2011;87:ii39eii43. doi:10.1136/ sti.2010.047712

9. Crowther-Gibson P, Govender N, Lewis DA, Bamford C, Brink A, von Gottberg A et al. Part IV: Human infections and antibiotic resistance. S Afr Med J.. August 2011;101(8):567-78.
10. Unemo M, Shafer WM. Antimicrobial Resistance in Neisseria gonorrhoeae in the 21st Century: Past, Evolution, and Future. Clin Microbiol Rev. 2014;27(93):587-613.

11. Krupp K, Madhivanan P. Antibiotic resistance in prevalent bacterial and protozoan sexually transmitted infections. Indian J Sex Transm Dis. January-June 2015;36(1):3-8. doi: 10.4103/0253-7184.156680

12. Erasmus L, Quan V, Hunt G, Radebe F. Introduction to GERMS-SA clinic based surveillance for TB, HIV and other STIs and related drug resistance. Communicable Diseases Surveillance Bulletin. June 2015 [cited 24 October 2016];13(2):46-8. Available from: http://www.nicd.ac.za/assets/files/ GERMS-SA\%20clinic\%20based\%20surveillance.pdf 


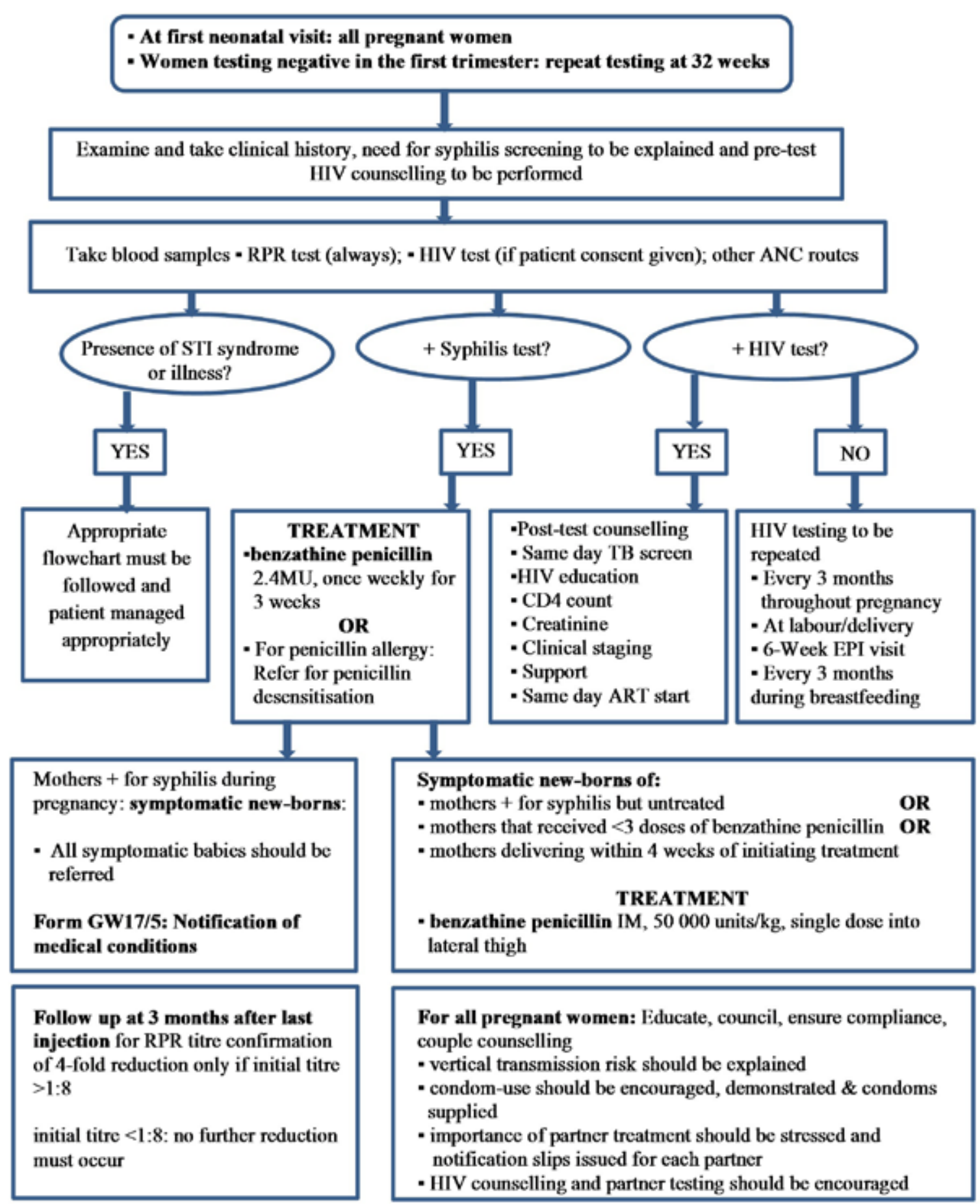

Figure 9: Flowchart of syndromic treatment of syphilis in pregnant women. (adapted from the revised syphilis (pregnancy) flowchart of the Primary Health Care Standard Treatment Guidelines and Essential Drugs List, 2015)23

13. Centres for Disease Control and Prevention. Antibiotic resistance threatens gonorrhoea treatment. 14 July 2016 [cited ]. Available from: http://www.cdc.gov/ nchhstp/newsroom/2016/gonorrhea-treatment-press-release.html

14. Centres for Disease Control and Prevention. CDC Fact sheet: Addressing the Threat of Drug-Resistant Gonorrhoea, July 2016 [cit]. Available from: http://www. cdc.gov/nchhstp/newsroom/docs/factsheets/drug-resistant-gonorrhea.pdf

15. WHO. Growing antibiotic resistance forces updates to recommended treatment for sexually transmitted infections. August 2016 [cit]. Available from: http:// www.who.int/mediacentre/news/releases/2016/antibiotics-sexual-infections/ en/.

16. Kularatne R, Maseko V, Gumede L, Radebe F, Kufa-Chakezha T. Neisseria gonorrhoeae antimicrobial resistance surveillance in Gauteng province,
South Africa. Communicable Diseases Surveillance Bulletin. September 2016;14(3):56-64.

17. WHO. WHO guidelines for the treatment of Treponema pallidum (syphilis). 2016 [cited ];1-50. Available from: http://apps.who.int/iris/bitstr eam/10665/249572/1/9789241549806-eng.pdf

18. Vuylsteke B. Current status of syndromic management of sexually transmitted infections in developing countries. Sex Transm Infect. 2004;80: 333-4. doi: 10.1136/sti.2004.009407

19. Low N, Broutet N, Adu-Sarkodie Y, Barton P, Hossain M, Hawkes S. Sexual and Reproductive Health 5: Global control of sexually transmitted infections. Lancet 2006; 368(9551):2001-16. 


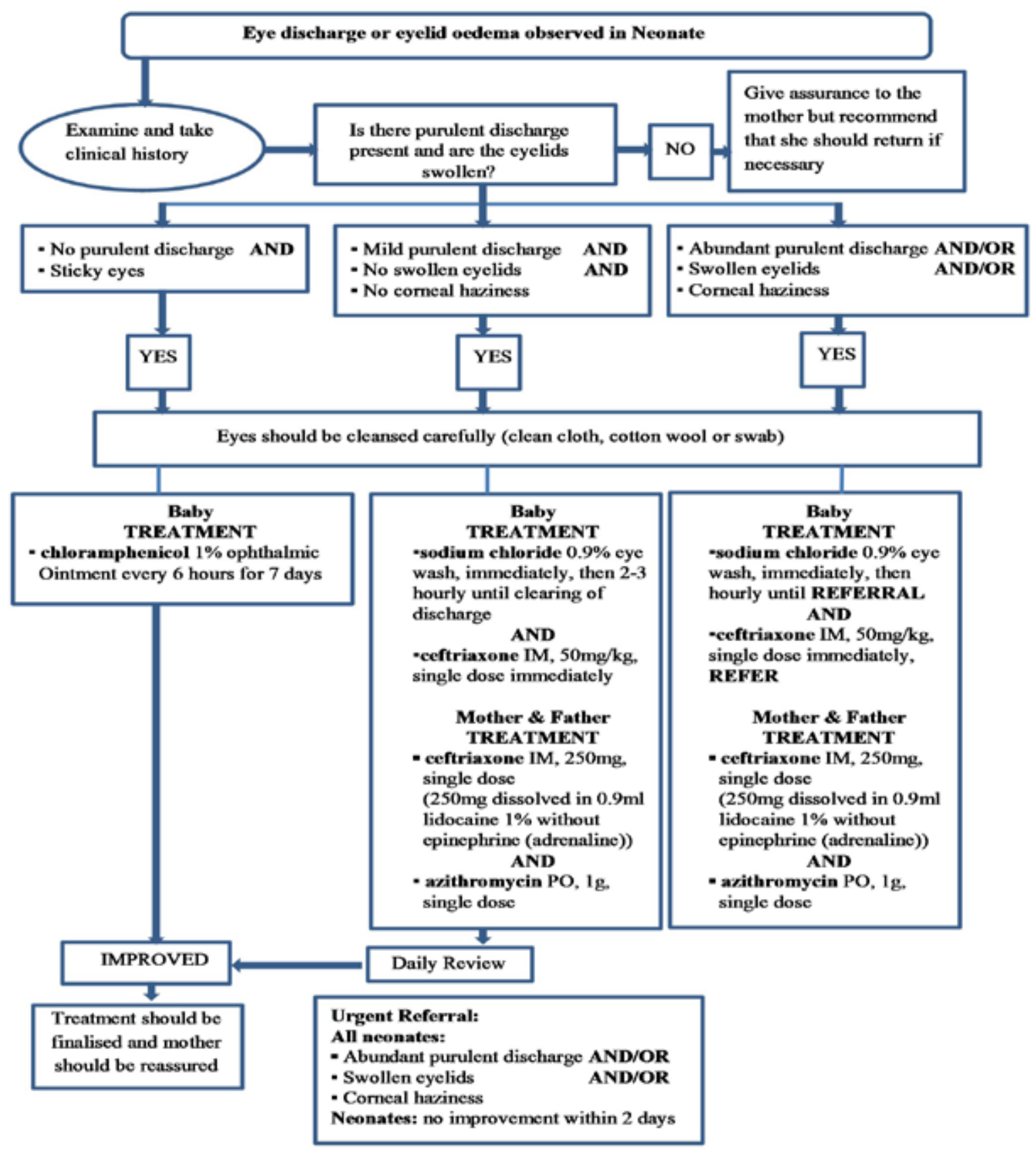

Figure 10: Flowchart of syndromic treatment of syphilis causing neonatal conjunctivitis. (adapted from the revised syphilis (neonatal) flowchart of the Primary Health Care Standard Treatment Guidelines and Essential Drugs List, 2015 ${ }^{23}$

20. Lewis DA, Marumo E. Revision of the national guideline for first-line comprehensive management and control of sexually transmitted infections: what's new and why? SA Fam Pract. 2010;52(1):20-4.

21. Workowski KA, Bolan GA. Centres for Disease Control and Prevention. Sexually Transmitted Diseases Treatment Guidelines. Recommendations and Reports 5 June 2015 [cited 29 September 2016];64(RR3);1-137. Available from: http://www. cdc.gov/mmwr/preview/mmwrhtml/rr6403a1.htm.

22. Workowski K. New STD Guidelines for 2015. Medscape. 5 Oct 2015 [cit]. Available from: http://www.medscape.com/viewarticle/851494

23. National Department of Health, South Africa. Sexually Transmitted Infections Management Guidelines 2015 [cited 29 September 2016]. Adapted from:
Standard Treatment Guidelines and Essential Drugs List PHC (2014) Available from: http://www.kznhealth.gov.za/family/STI-guidelines-2015.pdf

24. Roett MA, Mayor MT, Uduhiri KA. Diagnosis and Management of Genital Ulcers. Indian J Clin Prac. 2013;24(6):507-15.

25. Deivam S, Seethalakshmi RS, Balasubramanian N, Rajalakshmi R, Priyadharshini S, Lakshmi Priya P, et al. Case report of bubo - STD or non STD. Int J Dev Res. 2014;4(8):1787-9.

26. Arunkumar S, Murugan S, Sowdhamani B, Sureshkumar R. Balanitis and Balanoposthitis - Review article. Int J Res Health Sci [Internet]. 31 Jan 2014 [cited 24 October 2016];2(1):375-92. Available from: http://www.ijrhs.com/issues. php?val= Volume2\&iss =lssue 1 